AperTO - Archivio Istituzionale Open Access dell'Università di Torino

\title{
Application of 2 different hemostatic procedures during microsurgical median nerve reconstruction in the rat does not hinder axonal regeneration.
}

\section{This is the author's manuscript}

Original Citation:

Availability:

This version is available http://hdl.handle.net/2318/93288

since

Published version:

DOI:10.1227/NEU.0b013e3182127bc4

Terms of use:

Open Access

Anyone can freely access the full text of works made available as "Open Access". Works made available under a Creative Commons license can be used according to the terms and conditions of said license. Use of all other works requires consent of the right holder (author or publisher) if not exempted from copyright protection by the applicable law. 


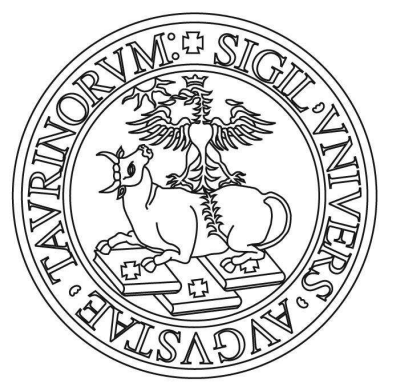

\section{UNIVERSITÀ DEGLI STUDI DI TORINO}

This is an author version of the contribution published on:

Neurosurgery, 68(5), 2011, DOI:10.1227/NEU.0b013e3182127bc4

Sinis $N$, Manoli $T$, Schiefer $J L$, Werdin $F$, Jaminet $P$, Kraus $A$, Fornaro $M$,

Raimondo S, Geuna S, Schaller HE, 68, Lippincot William\&Wilkins, 2011, 1399-1404

The definitive version is available at:

http://iournals./ww.com/neurosurgery/Abstract/2011/05000/Application of 2 Diffe rent Hemostatic Procedures.28.aspx 


\title{
Application of 2 different hemostatic procedures during microsurgical median nerve reconstruction in the rat does not hinder axonal regeneration
}

\begin{abstract}
Nektarios Sinis, MD* ${ }^{*}$; Theodora Manoli, MD*§; Jennifer Lynn Schiefer, MD§; Frank Werdin, MD§; Patrick Jaminet, MD§ Armin Kraus, MD§; Michele Fornaro, PhD; Stefania Raimondo, PhD; Stefano Geuna, MD; Hans-Eberhard Schaller, MD§ $\ddagger$ Department of Plastic Surgery, Martin-Luther Hospital, Berlin, Germany; §Department of Hand, Plastic, and Reconstructive Surgery with Burn Unit, BG Trauma Centre, University of Tuebin-gen, Tuebingen, Germany; \{Department of Clinical and Biological Sciences, University of Turin, San Luigi Hospital, Turin, Italy

*These authors have contributed equally contributed to this article.
\end{abstract}

\begin{abstract}
BACKGROUND: Hemostatic procedures by means of electrical coagulation or applica-tion of topical agents are widely used to avoid postoperative bleeding during micro-surgical reconstruction of peripheral nerves. It is speculated, however, that extensive hemostasis could provoke significant nerve damage.

OBJECTIVE: To examine the effects of 2 intraoperative hemostatic procedures on peripheral nerve regeneration.

METHODS: In 36 adult rats divided into 3 groups, the median nerve was transected and repaired by end-to-end neurorrhaphy. During surgery, bleeding was treated in groups 2 and 3 by application of either the topical hemostatic agent Floseal or routine bipolar coagulation. The degree of nerve regeneration was assessed in terms of motor function recovery using weekly grasping test evaluation for 3 months, muscle mass recovery of flexor digitorum sublimis, and stereological assessment of myelinated axon regeneration.

RESULTS: Neither of the 2 applied hemostatic methods induced any negative effects on nerve regeneration as defined by grip strength, muscle mass recovery, and morphology of myelinated nerve fibers. In contrast, Floseal-treated animals showed a faster progression of motor function recovery, and animals subjected to bipolar coagulation revealed a higher muscle mass recovery compared with the control group.
\end{abstract}

CONCLUSION: Controlled application of bipolar coagulation or Floseal does not exert a negative effect on the nerve regeneration outcome in rats. We propose that these methods can be applied safely to patients.

KEY WORDS: Blood coagulation, Hemostatic agent, Median nerve, Nerve reconstruction, Nerve regeneration, Rat

Peripheral nerve reconstruction is attracting more and more experimental and clinical research, and the progressive spread of microsurgical skills among surgeons worldwide is expected to maintain this trend over the near future.1,2 In this evolving scenario, basic science should provide the surgeon with as much information as possible about the various factors that might influence the clinical outcome of nerve surgery. This should include information not only on the possible tools to improve the regeneration process during and after surgery but also on the potential intentional effects or factors, which might depend on the surgical technique applied.

Among these factors, the use of bipolar coagulation to avoid bleeding might represent a possible source of damage to the nerve stumps because they can be unintentionally burned during the surgical act. Contrary to nerves treated by monopolar coagulation, application of bipolar coagulation was shown to preserve Schwann cell architecture and to cause less demyelination in previous studies.3,4 
An alternative to electric coagulation is the application of topical hemostatic agents. There are several hemostatic agents to choose from, and their number is increasing. The most common hemostatic agents are collagen, fibrin, and thrombin sealants. ${ }^{5}$ Floseal, a combination of a bo-vinederived gelatin-based matrix and a human-derived thrombin component, is an efficient topical hemostaticum that is being widely used during microsurgical procedures. ${ }^{6}$ Because the im-pact of hemostatic agents on nerve regeneration has not been specifically examined yet, one can speculate that they could negatively influence postoperative functional outcome.

The aim of the present study was to test whether unintentional nerve stump damage by bipolar coagulation or Floseal application exerts a negative effect on the outcome after end-to-end median nerve neurorrhaphy in the rat.

\section{METHODS}

\section{Surgery, Experimental Design, and Animal Surveillance}

Thirty-six Wistar adult female rats weighing 220 to $240 \mathrm{~g}$ each were used in this study. Good laboratory animal practice was observed according to the international standards for animal experimentation (EC Council Directive of November 1986, 86/609/EEC) and following approval by local government. Rats were fed standard laboratory food (Ssniff, Soest, Germany) and provided tap water ad libitum and an artificial light/dark cycle (12 hour light/12 hour dark). For the assessment of animal well-being, a careful daily animal surveillance was used to monitor passive and active movement, automutilation, skin ulcers, and joint contracture, especially during early postoperative times. In addi-tion, animal body weight was measured weekly.

Rats were operated on under deep intraperitoneal anesthesia with $100 \mathrm{mg} / \mathrm{kg}$ ketamine and 5 $\mathrm{mg} / \mathrm{kg}$ xylazine under clean conditions and with the aid of an operating microscope. The right median nerve was approached from the axillary region and then transected at a level where the brachial artery crosses the median nerve.

Animals were then divided into 3 experimental groups, each consisting of 12 rats. In group 1, the 2 median nerve stumps were immediately approached and repaired with a primary nerve suture in an end-to-end fashion by means of 2 epineural stitches of 10-0 monofilament nylon (Figure 1). In group 2, $20 \mathrm{~mL}$ Floseal was applied to each nerve stump before microsurgical repair by end-to-end neurorrhaphy. The volume was estimated according to the size of the rat median nerve. It was the

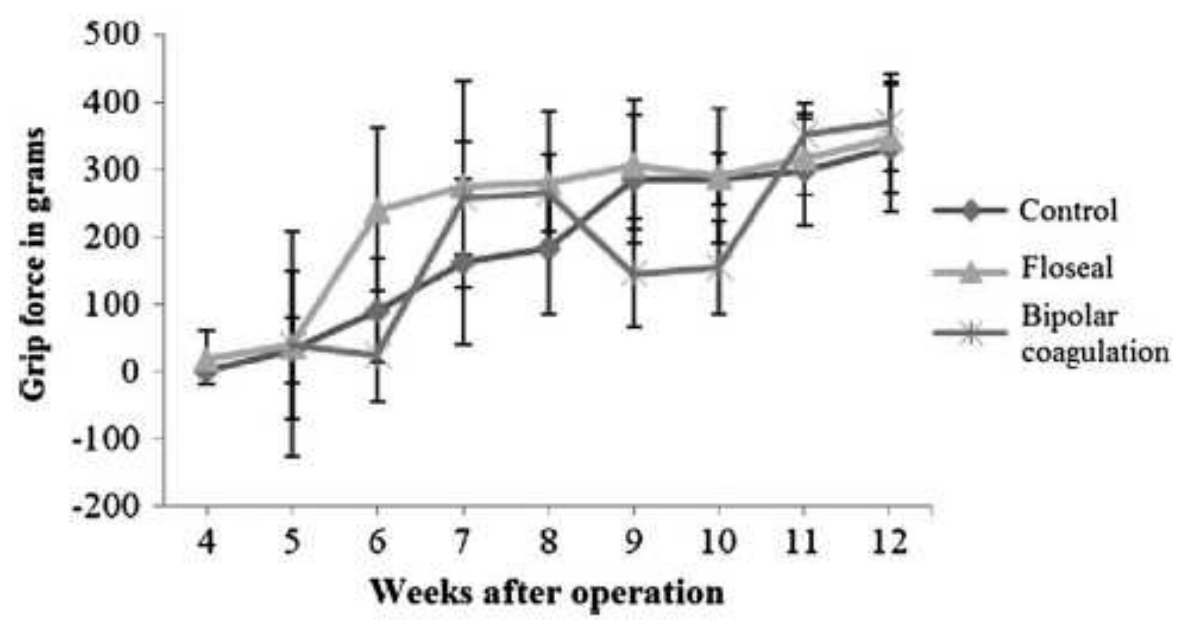

FIGURE 1. Comparison of motor function recovery assessed by the grasping test in the 3 experimental groups. 
amount of Floseal needed to flush the complete coaptation site with fluid because it is recommended that the entire bleeding site be kept in contact with the agent. Floseal is a combination of a bovine-derived gelatin-based matrix and a human-derived thrombin component. The gelatin com-ponent swells in the first 10 minutes up to $20 \%$ and hinders bleeding mechanically. Moreover, gelatin particles form a thrombin-rich surface on which secondary hemostasis occurs more effectively.7 Floseal is biocompatible and is completely resorbed in 6 to 8 weeks.

In group 3, the 2 nerve stumps were electrocoagulated briefly (for 1 second) by means of a microsurgical bipolar forceps. A programmed coagulation pattern with an autostop mechanism after 1 second of application time was used to standardize the electrical coagulation procedure at level 2 on the option board (Aesculap electrostimulator; output frequency, $450 \mathrm{kHz}$; modulation frequency, $1 \mathrm{kHz}$; power, $10 \mathrm{~W}$ ). The electrostimulation was chosen in accordance with human application with the same intensity. Because of the small size of the nerve, we chose to apply the stimulus with microforceps. The 2 median nerve stumps were then approached and repaired by end-to-end neurorrhaphy as described above. All operations were performed by a single experienced microsurgeon.

To ensure an unbiased evaluation of the results, all postoperative assessments were carried out by a blinded approach. Each rat was therefore given an identification code, so that at the time of evaluation, the investigators were unaware of the group to which the rat belonged.

\section{Assessment of Nerve Function Recovery}

Functional recovery during the first 12 postoperative weeks was measured by the grasping test.810 The grasping test was performed weekly beginning the first postoperative week. To obtain preoperative and postoperative comparisons within the data, all animals underwent the grasping test before surgery. Data were recorded and compared with the final results. The grasping test provides a quantitative assessment of finger flexion, which is controlled solely by the median nerve in the rat. The rat is gently lifted by the tail and allowed to grasp a grid connected to an ordinary electronic balance. While grasping, the animal continues to be lifted by the tail with increasing firmness until it loses its grip. At this precise moment, the value shown by the balance is recorded. After 3 grasping attempts, the highest value in grams displayed on the electronic balance is recorded and considered to reflect the maximum grip force for that specific animal at the respective experimental time point. To avoid grasping via the left (intact) paw, claws are covered by a piece of adhesive tape before testing.11

\section{Assessment of Muscle Weight}

Three months after surgery, all animals were anesthetized again with $100 \mathrm{mg} / \mathrm{kg}$ ketamine and 5 $\mathrm{mg} / \mathrm{kg}$ xylazine. The flexor digitorum sublimis muscle is one of the forearm muscles in rats that are exclusively innervated by the median nerve. The right flexor digitorum sublimis muscle was carefully exposed, withdrawn, and immediately weighed.

\section{Histological and Stereological Analyses of Nerve Fiber Regeneration}

For histological and stereological quantitative analyses of regenerated nerve fibers, 1-cm-long median nerve segments were harvested imme-diately distally to the suture site and fixed by immediate immersion in $2.5 \%$ purified glutaraldehyde and $0.5 \%$ saccarose in $0.1 \mathrm{~mol} / \mathrm{L}$ Sorensen phosphate buffer for 6 to 8 hours. Specimens were then washed in a solution containing 1.5\% saccarose in $0.1 \mathrm{~mol} / \mathrm{L}$ Sorensen phosphate buffer, postfixed in $1 \%$ osmium tetroxide, dehydrated, and embedded in resin. From each nerve, 2-mm-thick series of semithin transverse sections were cut starting from the distal stump of each median nerve specimen with an Ultracut UCT 
ultramicrotome (Leica Microsystems, Wetzlar, Germany) and stained with toluidine blue for highresolution light microscopy examination. On 1 randomly selected section from each nerve specimen, design-based stereological analysis was carried out by a DM4000B microscope equipped with a DFC320 digital camera and an IM50 image manager system (Leica Microsystems). The final mag-nification was 36.6 , enabling accurate identification and analysis of myelinated nerve fibers. On the randomly selected section, the total cross-sectional area of the nerve was measured, and then 12 to 16 sampling fields were selected through the use of a systematic random sampling protocol.12 In each sampling field, a 2-dimensional disector procedure, which is based on sampling the "tops" of fibers, was adopted to avoid the edge effect.13 Mean fiber density was then calculated by dividing the total number of nerve fibers $(n)$ within the sampling field by its area $(n / m m 2)$. Total fiber number was finally estimated by multi-plying the mean fiber density by the total cross-sectional area of the whole-nerve cross section.

Two-dimensional dissection probes were also used to select an un-biased representative sample of myelinated nerve fibers for measuring the following parameters: circle-fitting diameter of fiber (D) and axon (d), myelin thickness [( $D 2 d$ ) / 2], and axon/fiber diameter ratio, the $g$ ratio (d/D). The precision of the estimates was evaluated by calculating the coefficient of error as previously described.13,14 The sampling scheme was designed to keep the coefficient of error below 0.10 , which ensures enough accuracy for neuromorphological studies.15

\section{Statistical Analysis}

Statistical analysis of quantitative data obtained by the grasping test was carried out with 1-way repeated-measures analysis of variance over time. When significant differences were detected by repeated-measures analysis of variance, posthoc multiple pairwise comparisons were carried out with the Student-Newman-Keuls test. Statistical analysis of quantitative data on muscle weight and axon number and density was carried out with 1-way ANOVA. Statistical significance was established as $\mathrm{P}, .05$.

\section{Results}

Animal surveillance showed that animal well-being was maintained during all postoperative times. All animals survived until the end of the experiment, 3 months after operation.

Figure 1 shows the results of the weekly grasping test assess-ment. In all experimental groups, functional recovery started between postoperative weeks 4 and 6 and increased progressively until the end of experiments at postoperative week 12, when it reached $80 \%$ of preoperative baseline values. In general, Floseal-treated animals (group 2) showed a significantly faster progression of motor function recovery $(P, .05)$ than animals treated by no hemostatic method (group 1$)$ or by bipolar coagulation (group 3). The final function recovery at month 3 postoperatively showed no statistically significant difference between the 3 experimental groups $(P$. .05).

The mean masses of reinnervated flexor digitorum sublimis muscles in the 3 experimental groups are given with their SDs in Table 1. When t tests between the 3 experimental groups were 


\begin{tabular}{|c|c|}
\hline Group & $\begin{array}{c}\text { Wet Muscle } \\
\text { Weight, } \mathbf{m g}^{*}\end{array}$ \\
\hline 1 & $118 \pm 21.0$ \\
\hline 2 & $127 \pm 20.0$ \\
\hline 3 & $140 \pm 15.5$ \\
\hline
\end{tabular}

*Values are mean 6 SD.

TABLE 1. Comparison of Muscle Mass (Mean Weight) of Reinnervated Flexor Digitorum Muscles in the 3 Experimental Groups

performed, a significantly higher muscle mass in animals treated by bipolar coagulation (group 3) was found compared with the control group 1 ( $P, .05)$. There was no statistically significant difference between the Floseal-treated group 2 and bipolar coagulation-treated group 3 (P . .05).

The morphological appearance of regenerated nerve fibers in the 3 experimental groups is shown in Figure 2. No qualitative morphological differences could be seen among the different groups. Design-based quantitative assessment of regenerated axons (Table 2) confirmed the qualitative analysis because sta-tistical comparison revealed no significant intergroup differences for any of the morphometric parameters investigated ( $P$. .05).

\section{Discussion}

Hemostatic applications in the form of topical agents or electrocoagulation are a common practice during surgery of peripheral nerves. Especially at the level of brachial plexus nerves, intraoperative bleeding caused by injury of accompanying, relatively large-caliber nutritive vasa nervorum is not a rare phenomenon. In that situation, hemostatic procedures have to be applied to avoid obscuration of the operative field. However, the effects of such methods have not, to the best of our knowledge, been investigated in a controlled experimental setting. It can be postulated that the reduction of intraoperative and postoperative bleeding could have a positive impact on the quality of peripheral nerve regeneration. On the other hand, electrocoagulation or application of hemostatic agents could provoke damage to the nerve stumps, which might interfere with axonal regeneration and lead to a delayed or insufficient outcome after peripheral nerve neurorraphy. It has already been shown that monopolar co-agulation provoked significant Schwann cell destruction com-pared with bipolar coagulation and should therefore be avoided when working around nerves.3,4

The present study aimed at comparatively investigating the effects of intraoperative bipolar electrocoagulation and applica-tion of the topical hemostaticum Floseal on nerve fiber re-generation after end-to-end neurorrhaphy in rats. Results revealed that neither of the 2 applied hemostatic methods induced any negative effects on nerve regeneration as defined by grip strength, muscle mass recovery, and morphology of myelinated nerve fibers 

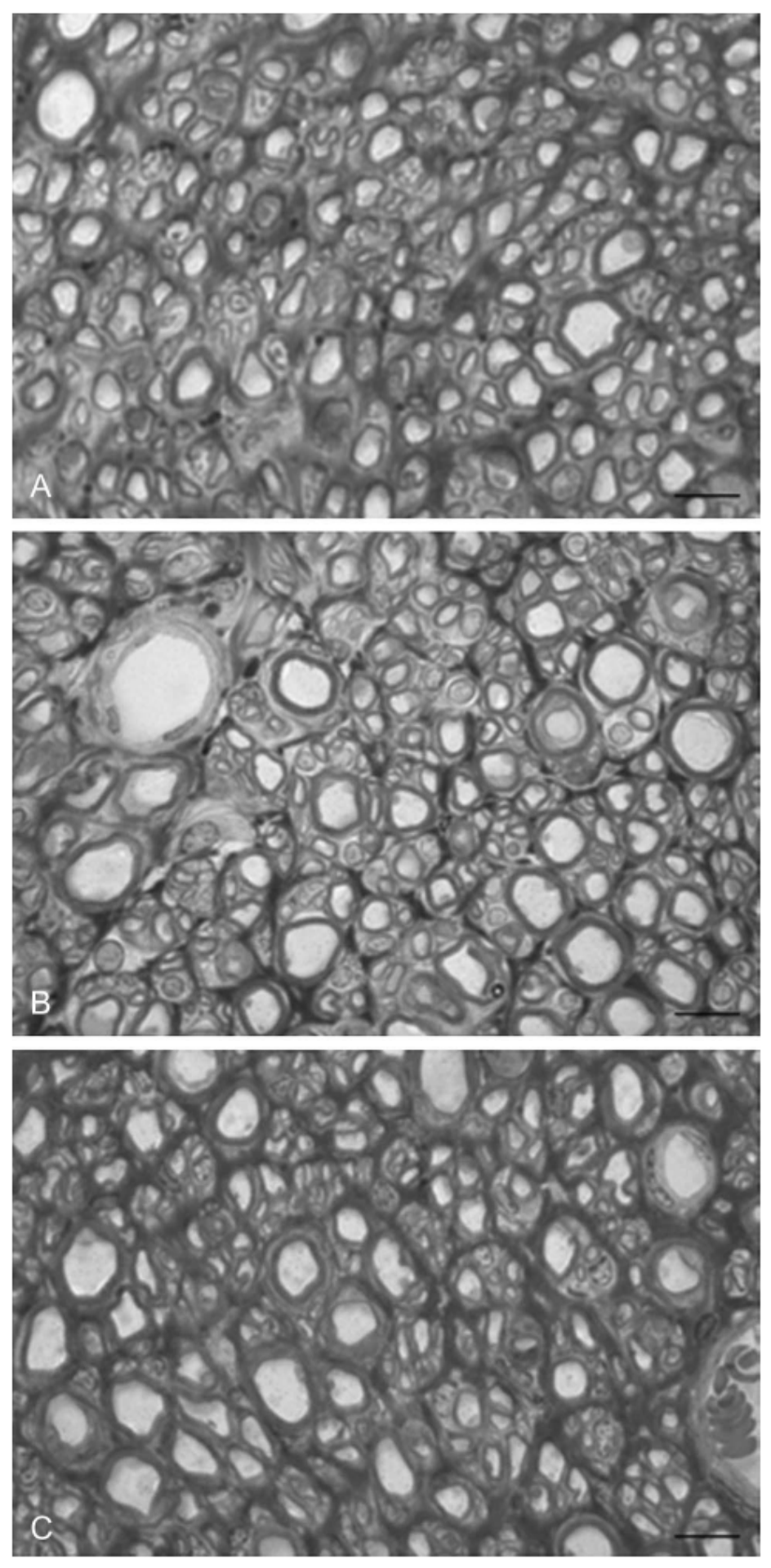

FIGURE 2. Light microscope toluidine blue-stained micrographs of the re-generated median nerve in the 3 experimental groups: A, group 1; B, group 2; C, group 3. Magnification, 32000.

as predictors of recovery. In contrast, Floseal-treated animals showed a faster progression of motor function recovery, whereas animals subjected to bipolar coagulation revealed a higher muscle mass recovery compared with the control group. One possible reason for the positive effects of the 2 tested hemostatic methods 


\begin{tabular}{|c|c|c|c|c|}
\hline \multicolumn{5}{|c|}{ Fiber Diameter, Myelin } \\
\hline Group & Fibers, $\mathbf{n}$ & $\boldsymbol{\mu m}^{\mathrm{a}}$ & Thickness, $\boldsymbol{\mu m}^{\mathrm{a}}$ & gRatio \\
\hline 1 & $6829 \pm 1430$ & $3.65 \pm 0.28$ & $0.46 \pm 0.07$ & $0.73 \pm 0.06$ \\
\hline 2 & $7798 \pm 1698$ & $3.86 \pm 0.28$ & $0.44 \pm 0.03$ & $0.76 \pm 0.01$ \\
\hline 3 & $7679 \pm 507$ & $3.56 \pm 0.18$ & $0.45 \pm 0.04$ & $0.74 \pm 0.02$ \\
\hline
\end{tabular}

${ }^{a}$ Values are mean 6 SD.

TABLE 2. Comparison of Stereological Predictors of Nerve Fiber Regeneration in the Regenerated Median Nerve: Total Number of Fibers, Mean Fiber Diameter, Myelin Thickness, and g Ratio

on nerve regeneration parameters could be the reduction of postoperative loss of nutrients by bleeding. An adequate nutrient supply to regenerating axons and Schwann cells through intrafascicular and epineural vessels is important for effective nerve regeneration.16 The faster progression of motor function recovery in Floseal-treated animals is not surprising because no negative effects of the local hemostatic agent on nerve regeneration are expected. Moreover, faster axon growth in a high-viscosity gel matrix applied between the 2 nerve stumps could be postulated.17

On the other hand, bipolar coagulation can cause thermal damage to Schwann cells, which can compensate for or even overwhelm the positive hemostatic effect. This could explain the delayed progression of motor recovery at week 6 after operation. It is important to illustrate that in this study bipolar coagulation was applied by microsurgical forceps and a programmed co-agulation pattern with an autostop mechanism after 1 second of application time. When bigger macroscopic forceps are used, the area of thermocoagulation could be unpredictably big in small nerves, provoking significant nerve damage. Moreover, it cannot be excluded that longer coagulation times or different coagulation intensities than those used in this study may have a negative impact on functional and morphological results of nerve regeneration. However, because the potential for axonal regeneration after injury is different in rodents than in humans, it is difficult to transfer the settings of bipolar intensity that gen-erated satisfactory results to human surgery. Nevertheless, these results demonstrate, in accordance with clinical observations, that bipolar coagulation does not cause harm to nerves if applied carefully.

In addition, the results raise the question of whether axons can preserve their regenerative potential after extensive thermal damage during microsurgical reconstruction. Further research should focus on the hypothesis that a postconditioning effect like bipolar coagulation of the nerve stumps after neurotmesis and before neurorraphy can stimulate the regenerative potential of transected axons in the proximal nerve stump as well as wallerian degeneration in the distal stump. It was recently shown, for instance, that electrostimulation induces cell differentiation of fibroblasts.18 These effects could also be visualized by observation of early changes and effects of regeneration during the first hours after application of electrocautery. Because our results showed a higher muscle mass recovery in animals treated by bipolar coagulation than in the control group, it is speculated that neuromuscular repair might have been improved by a post-conditioning mechanism. However, this question could also be addressed in an electrophysiological study in which the denervation and reinnervation of the muscle could be demonstrated according to time and place (ie, different parts of the muscle).

Our study suggests that bipolar coagulation or application of local hemostatic agents during 
microsurgical nerve reconstruction does not harm axon regeneration. Although implications of clinical applications obtained through experimental observations in laboratory animal models should be critically considered, the results of the present study support the view that the examined hemostatic methods can be used safely in patients.

\section{Conclusion}

Controlled application of the local hemostatic agent Floseal or bipolar coagulation of the nerve stumps did not hinder nerve regeneration after end-to-end neurorraphy of the median nerve in the rat. Floseal-treated animals showed a faster motor recovery than the control group.

\section{Disclosure}

This work was supported by a Fortune $(01 \mathrm{H} 2 \mathrm{~S})$ grant from the University of Tuebingen and by grants from the Regione Piemonte (Ricerca Sanitaria Finalizzata) and the Italian Ministry of University and Research. The authors have no personal financial or institutional interest in any of the drugs, materials, or devices described in this article.

\section{References}

1.Evans GR. Peripheral nerve injury: a review and approach to tissue engineered constructs. Anat Rec. 2001;263(4):396-404.

2.Lundborg G. A 25-year perspective of peripheral nerve surgery: evolving neuroscientific concepts and clinical significance. J Hand Surg Am. 2000;25(3): 391-414.

3.Zohar Y, Sadov R, Strauss M, Djialdetti M. Ultrastructural study of peripheral nerve injury induced by monopolar and bipolar diathermy. Ann Otol Rhinol Laryngol. 1996;105(9):673-677.

4.Schietroma JJ, Tenzel RR. The effects of cautery on the optic nerve. Ophthal Plast Reconstr Surg. 1990;6(2):102-107.

5.Barnard J, Millner R. A review of topical hemostatic agents for use in cardiac surgery. Ann Thorac Surg. 2009;88(4):1377-1383.

6. Oz MC, Cosgrove DM III, Badduke BR, et al. Controlled clinical trial of a novel hemostatic agent in cardiac surgery: the Fusion Matrix Study Group. Ann Thorac Surg. 2000;69(5):1376-1382.

7.Stassen JM, Arnout J, Deckmyn H. The hemostatic system. Curr Med Chem. 2004;11(17):22452260.

8.Bertelli JA, Mira JC. The grasping test: a simple behavioral method for objective quantitative assessment of peripheral nerve regeneration in the rat. J Neurosci Methods. 1995;58(1-2):151155.

9.Papalia I, Tos P, Stagno d'Alcontres F, Battiston B, Geuna S. On the use of the grasping test in the rat median nerve model: a re-appraisal of its efficacy for quantitative assessment of motor function recovery. J Neurosci Methods. 2003;127(1):43-47.

10. Sinis N, Schaller HE, Schulte-Eversum C, et al. Nerve regeneration across a 2-cm gap in the rat median nerve using a resorbable nerve conduit filled with Schwann cells. J Neurosurg. 2005;103(6):1067-1076.

11. Ronchi G, Nicolino S, Raimondo S, et al. Functional and morphological as-sessment of a standardized crush injury of the rat median nerve. J Neurosci Methods. 2009;179(1):51-57.

12. Piskin A, Kaplan S, Aktas A, et al. Platelet gel does not improve peripheral nerve regeneration: an electrophysiological, stereological, and electron microscopic study. Microsurgery. 2009;29(2):144-153.

13. Raimondo S, Fornaro M, Di Scipio F, Ronchi G, Giacobini-Robecchi MG, Geuna S. Chapter 5: methods and protocols in peripheral nerve regeneration experimental research, part II: morphological techniques. Int Rev Neurobiol. 2009;87:81-103.

14. Schmitz C. Variation of fractionator estimates and its prediction. Anat Embryol (Berl). 1998;198(5):371-397.

15. Pakkenberg B, Gundersen HJ. Neocortical neuron number in humans: effect of sex and age. 
J Comp Neurol. 1997;384(2):312-320.

16. Bell MA, Weddell AG. A descriptive study of the blood vessels of the sciatic nerve in the rat, man and other mammals. Brain. 1984;107 (pt 3):871-898.

17. Nakayama K, Takakuda K, Koyama Y, et al. Enhancement of peripheral nerve regeneration using bioabsorbable polymer tubes packed with fibrin gel. Artif Organs. 2007;31(7):500-508.

18. Genovese JA, Spadaccio C, Langer J, Habe J, Jackson J, Patel AN. Electro-stimulation induces cardiomyocyte predifferentiation of fibroblasts. Biochem Biophys Res Commun. 2008;370(3):450-455. 